\title{
The Study on the Corrosion Behavior of Alloy Materials in Phase Change Materials (PCMs)
}

\author{
Handongfei $^{1, a}$, Chendianbin ${ }^{2, b}$ \\ 1. 2 Baicheng ordnance test center of China, Baicheng,qingJilin, 137001, China \\ a7289734@qq.com bsunshinesun2016@163.com
}

Keywords: Alloy; phase change materials (PCMs); corrosion; stainless steel

\begin{abstract}
Due to huge advantages, phase change materials (PCMs) are widely applied to various fields. Particularly, they are essential materials in regeneration, development and utilization of energy. However, because PCMs generally contain alloy materials and have some corrosivity, some leakage losses may be suffered by PCMs, driving people to strengthen the research concern on the corrosion behavior of alloy materials in PCMs. In the paper, the author regards inorganic alloy PCMs in PCMs as the research emphasis, selects six alloy materials, including red copper, brass, 5052 aluminum, 6063 aluminum, 202 stainless steel and 304 stainless steel to do the experimental study, and explores from corrosion rate, corrosion products, morphology and energy of corrosion products. It shows that the corrosion resistance of six alloy materials in PCMs is ranked as: 304 stainless steel $>202$ stainless steel $>6063$ aluminum alloy $>5052$ aluminum alloy $>$ brass $>$ red copper, providing some theoretical basis on studying corrosion prevention of PCMs in the future.
\end{abstract}

\section{Brief Introduction}

PCMs can store thermal energy in the phase change process, so as to solve a problem of unmatched time and space for thermal energy and greatly reduce environmental pollution. PCMs are main materials to improve energy efficiency and develop renewable energy sources and are widely applied in different fields. According to differences of phase change forms, PCM materials can be divided into solid-solid PCMs and solid-liquid PCMs, liquid-gas PCMs and solid-gas PCMs. The commonest one is the solid-liquid PCMs. PCMs also can be divided into inorganic PCMs(crystal hydrous salts, metals and alloys, etc), organic PCMs(paraffin and stearic acid), inorganic/organic conforming PCMs(as shown in Table 1). The further studies find that liquid-solid inorganic PCMs have the large density, the large latent heat of phase change and small volume changes, etc. Thus, PCMs become the stored energy media with the extensive applied prospects, but inorganic PCMs are mainly made by multiple inorganic salts and have the strong corrosivity on sealed PCMs. In practical applications, containers are often conducted corrosion perforation, resulting in huge losses in leaking energy storage materials. It must be concerned in current days.

Tab. 1 Phase Change Temperature and Enthalpy of Phase Change for Organic/Inorganic Conforming FSPCMs

\begin{tabular}{|c|c|c|c|}
\hline & $\begin{array}{c}\text { Contents of } \\
\text { PCMs }(\%)\end{array}$ & $\begin{array}{c}\text { Phase Change } \\
\text { Temperature }\left({ }^{\circ} \mathrm{C}\right)\end{array}$ & $\begin{array}{c}\text { Enthalpy of Phase } \\
\text { Change }(\mathrm{kJ} \cdot \mathrm{kg})\end{array}$ \\
\hline Paraffin/Polyethylene & 75 & 58 & 160 \\
\hline Stearic Acid/SiO 2 & $49-62$ & 60 & $25.68-195.80$ \\
\hline Paraffin/SBS & $55=60$ & 57 & $81.70-165.20$ \\
\hline
\end{tabular}

\section{The Study About High-temperature Inorganic PCMs}

Considering the unique advantages of PCMs, it has the important significance on energy development and utilization. It is mainly concentrated on fuse salts and metal alloys in high-temperature PCMs. PCMs mainly have the following advantages, including high latent heat of phase change, large heat conductivity coefficient and small phase change volume, so they are 
widely applied in some fields, including solar thermal power plants, electric power, space stations and industrial waste heat, etc.

The studies on PCMs find that the heat conductivity coefficient of nitrate is lower than $0.5 \mathrm{~W} /(\mathrm{m} \cdot \mathrm{K})$, requiring for the longer time as heat release or the greater temperature difference as heat release. The heat conductivity coefficient of metals is large and even can reach hundreds of times by comparing with organic PCM. As a result, heat-transfer capability of metals is extremely good. The corresponding device volume of energy storage and heat exchange is also small. For example, $\mathrm{Al}, \mathrm{Cu}, \mathrm{Mg}, \mathrm{Si}$ and $\mathrm{Zn}$, etc., generally have the phase change temperature from $700 \mathrm{~K}$ to $900 \mathrm{~K}$. The heat conductivity coefficient is high and latent heat of phase change is large. As a result, these are primary metal PCMs. The specific situation is shown in Table 2.

Tab.2 Thermal Performance of Some Metal and Alloyed PCMs

\begin{tabular}{|c|c|c|}
\hline Substances (Mass Fraction/\%) & Melting Temperature $/{ }^{\circ} \mathrm{C}$ & Melting Heat $/(\mathrm{kJ} / \mathrm{kg})$ \\
\hline $\mathrm{Li}$ & 181 & 435 \\
\hline $\mathrm{Al}$ & 660 & 398 \\
\hline $\mathrm{Cu}$ & 1083 & 205 \\
\hline $\mathrm{Ca}$ & 848 & 218 \\
\hline $\mathrm{Mg}$ & 650 & 401 \\
\hline $46.3 \mathrm{Mg}-53.7 \mathrm{Zn}$ & 340 & 185 \\
\hline $96 \mathrm{Zn}-4 \mathrm{Al}$ & 381 & 138 \\
\hline 34.65Mg-65.35Al & 497 & 285 \\
\hline $60.8 \mathrm{Al}-33.2 \mathrm{Cu}-6.0 \mathrm{Mg}$ & 506 & 365 \\
\hline $64.1 \mathrm{Al}-5.2 \mathrm{Si}-28 \mathrm{Ci}-2.2 \mathrm{Mg}$ & 507 & 374 \\
\hline $68.5 \mathrm{Al}-5.0 \mathrm{Si}-26.5 \mathrm{Cu}$ & 525 & 364 \\
\hline $66.92 \mathrm{Al}-33.08 \mathrm{Cu}$ & 548 & 372 \\
\hline $83.14 \mathrm{Al}-11.7 \mathrm{Si}-5.16 \mathrm{Mg}$ & 555 & 485 \\
\hline $87.76 \mathrm{Al}-12.24 \mathrm{Si}$ & 557 & 498 \\
\hline $46.3 \mathrm{Al}-4.6 \mathrm{Si}-49.1 \mathrm{Cu}$ & 571 & 406 \\
\hline $86.4 \mathrm{Al}=9.4 \mathrm{Si}-4.2 \mathrm{Sb}$ & 471 & 471 \\
\hline
\end{tabular}

In order to further know about the corrosion behavior of alloy materials in PCMs, the weight loss method is often applied the common metals, such as red cooper, brass, 5052 aluminum, 6063 aluminum, 202 stainless steel and 304 stainless steel, etc., to discuss their corrosion mechanism.

\section{Experimental Materials Instrument Preparations}

Experimental materials include $\mathrm{LiNO}_{3}$ and $\mathrm{Mg}\left(\mathrm{NO}_{3}\right)_{2} \cdot 6 \mathrm{H}_{2} \mathrm{O}$ which are analytical pure(TCM Group Chemical Reagent Co., Ltd) without purification of recrystallization. Alloy materials include red copper, brass, 5052 aluminum, 6063 aluminum, 202 stainless steel and 304 stainless steel. The thickness is about $1 \mathrm{~mm}$. The linear cutting machine is applied to cut materials into the specification of $50 \mathrm{~mm} \times 10 \mathrm{~mm}$. Components of alloy metals are different. Red copper contains $99.70 \%$ of $\mathrm{Cu}$; Brass contains $\mathrm{Cu}$, Zn, Fe, Ni and $\mathrm{Mn}$; 5052 aluminum contains $0.08 \%$ of $\mathrm{Cu}, 96.79 \%$ of $\mathrm{Al}, 2.40 \%$ of $\mathrm{Mg}, 0.35 \%$ of $\mathrm{Fe}, 0.17 \%$ of $\mathrm{Cr}$ and $0.21 \%$ of $\mathrm{Si} ; 6063$ Aluminum contains $\mathrm{Cu}, \mathrm{Al}, \mathrm{Zn}, \mathrm{Mg}, \mathrm{Fe}$ and Si; 202 stainless steel and 304 stainless steel have the same chemical components, but contents have some differences. For example, 202 stainless steel has Bal. of Fe(remaining components), $4-6 \%$ of $\mathrm{Ni}, 17-19 \%$ of $\mathrm{Cr},<1 \%$ of $\mathrm{Si}$, and $7.5-10 \%$ of Mn. Elements of 304 stainless steel contain 
Bal., 8.0-10.5\%, 18-20\%, $\leq 1 \%$, and $\leq 2.0 \%$, respectively. Instruments used in the experiment include BSA224S - CW type, T14101-L-type precision electronic balance, D/Max 2500-type X-ray diffractometer(XRD), Quanta-200-type environmental scanning electron microscope(E-SEM).

\section{Experimental Process and Result Analysis}

After preparing relevant materials and instruments, the experimental steps of weight loss method are applied to do an experiment, so as to know about the corrosivity of alloy materials in PCMs.

First of all, alloy materials are polished. Polished material samples are rinsed, dried and cooled. $\pm 0.1 \mathrm{mg}$ of precision electronic balance is applied to weigh for standby. Secondly, PCMs are prepared. $672 \mathrm{gLiNO}_{3}$ and $4128 \mathrm{gMg}\left(\mathrm{NO}_{3}\right)_{2} \cdot 6 \mathrm{H}_{2} \mathrm{O}$ reagents are applied. After heating and stirring sufficiently, they are placed in $72100 \mathrm{ml}$ of Teflon plastic tubes to reach $2 / 3$ of the tube volume. Thirdly, alloy materials in the experiment are placed PCMs for sealing and placed in $\pm 0.1^{\circ} \mathrm{C}$ of thermostatted pot at $75^{\circ} \mathrm{C}$ for soaking about $7,14,21,28 \mathrm{~d}$, respectively. Corrosives are grazed by using a brush. The acid wash prepared by JB/T7901-1999 Even Uniform Corrosion is used to do acid pickling, rinsed and dried for standby. Finally, alloy materials after being corroded for 28d are selected. XRD is applied to observe and analyze components of corrosion products. E-SEM is applied to scan microcosmic corrosion morphology. The EDS attached by SEM is applied to analyze components of corrosion products.

In the result analysis process, the computational formulas will be applied, including:

$$
D_{\text {失重 }}=\Delta \mathrm{m} /(S \bullet \rho)
$$

In the formula, $\mathrm{D}_{\text {weight loss }}$ refers to the thickness of the corrosion layer. $\Delta \mathrm{m}$ stands for the quality on the corrosion layer. $\mathrm{S}$ is the surface area of samples. $\rho$ is the mass density of experimental materials. According to the corrosion rate calculated by the formula, as shown in the formula (2), $t$ is the corrosion time.

$$
V_{\text {失重 }}=\Delta \mathrm{m} /(S \bullet \mathrm{t})
$$

Formulas (1) and (2) are combined and settled. The formula that computing alloy corrosion rate is finally obtained:

$$
\mathrm{v}=\left(\mathrm{m}_{0}-\mathrm{m}_{\mathrm{t}}\right) / \mathrm{St}
$$

$\mathrm{m}_{0}$ refers to the mass before alloy corrosion. $\mathrm{m}_{\mathrm{t}}$ is the alloy mass after removing corrosion products.

The experiment shows that the corrosion phenomenon of brass and red copper is relatively obvious. The corrosive hole is clear. After soaking for $28 \mathrm{~d}, \Delta \mathrm{m}$ reaches $21.3 \mathrm{mg}$. The mass change is large. E72 solution color of containing these two alloys becomes light blue. Thus, it can be observed that cupric ion is dissolved in the PCMs. $\Delta \mathrm{m}$ of 5052 aluminum is $1.7 \mathrm{mg}$, but there is the obvious pitting hole on the surface. For other three alloy materials, 6063 aluminum, 202 and 304 stainless steel have the smooth surface. There is no corrosion phenomenon with the naked eyes. After soaking, $\Delta \mathrm{m}$ has the small change, which can be neglected. The corrosive curve of 6 alloy materials in PCMs is shown in Figure 1. 


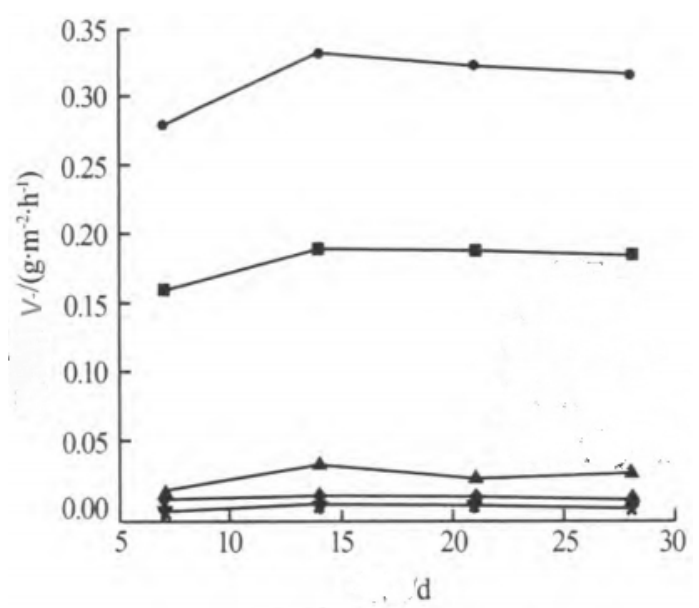

Notes: $\rightarrow$ is red copper, $\rightarrow$ is brass, $\rightarrow$ is 5052 aluminum, $\rightarrow$ is 6063 aluminum $\boldsymbol{\sim}^{-}$is 202 stainless steel $*$ is 304 stainless steel

Fig. 1: The Corrosion Rate Curve of 6 Alloy Materials

It can be observed from the above-mentioned curve, the corrosive rate is ranked from large to small as: red copper $>$ brass $>5052$ aluminum $>6063$ aluminum $>202$ stainless steel $>304$ stainless steel. By comparing with the corrosion rate , it can be observed that mass of red copper's corrosion products reaches $2803.2 \mathrm{~g} / \mathrm{m}^{2} ; 1576.8 \mathrm{~g} / \mathrm{m}^{2}$ for brass, 5052 aluminum alloy loss is not obviously, but pitting hole may bore a hole for containers. As a result, it may not suggest applying brass, red copper, and 5052 aluminum alloy for containers of E72 PCMs.

The components of corrosion products are further analyzed. XRD is applied to analyze the phase composition of corrosion products. Basically speaking, there is no miscellaneous peak. It has the obvious peak pattern and is identical to the standard spectrum diagram. Main corrosion products of red copper and brass are $\mathrm{Cu}_{2} \mathrm{O}$. Corrosion products of two aluminum alloys are $\mathrm{Al}_{2} \mathrm{O}_{3}$. In the XRD spectrum diagram of stainless steel, there is no characteristic peak of oxide, showing there is no corrosion product. The specific spectrum diagram is shown in Figure 2:
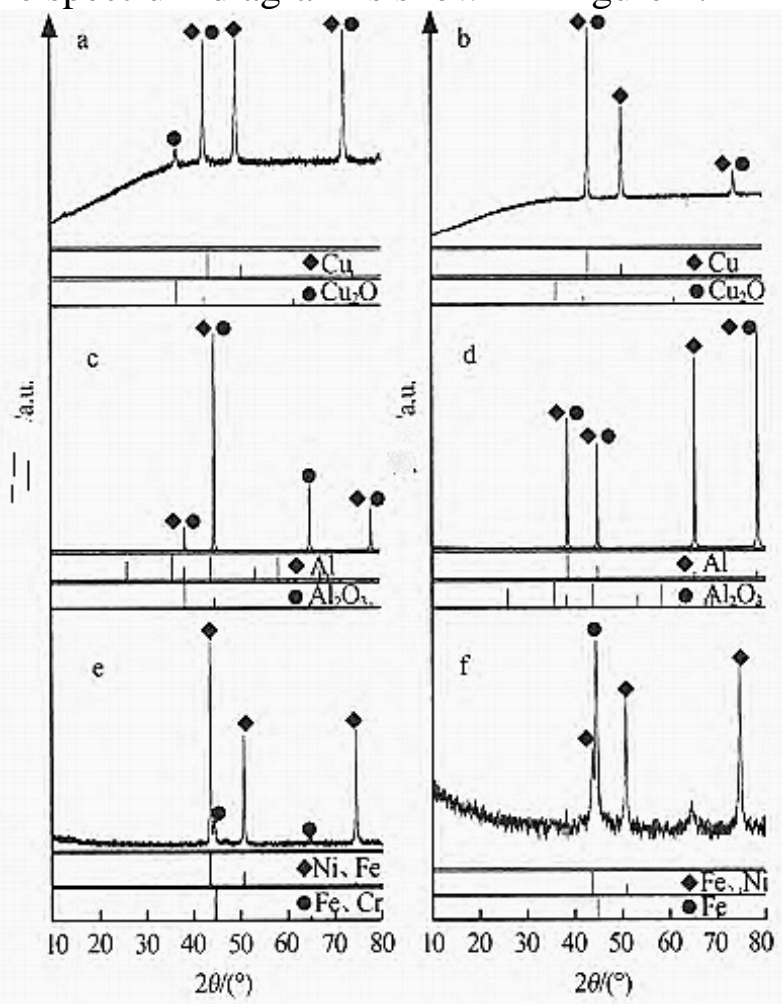

Notes: a, b, c, d, e, f stand for red copper, brass, 5052 aluminum, 6063 aluminum, 202 stainless steel and 304 stainless steel, respectively

Fig. 2: Corrosion Product XRD Spectrum Diagram of 6 Alloy Materials in E72 PCMs 
After knowing about corrosion products of 6 alloy materials in PCMs, scanning electron microscope is further applied to observe corrosion morphology and products in details. Corrosion samples are conducted EDS. The specific detection results are shown in Figure 3 and Figure 4(notes: stand for red copper, brass, 5052 aluminum, 6063 aluminum, 202 stainless steel and 304 stainless steel, respectively):
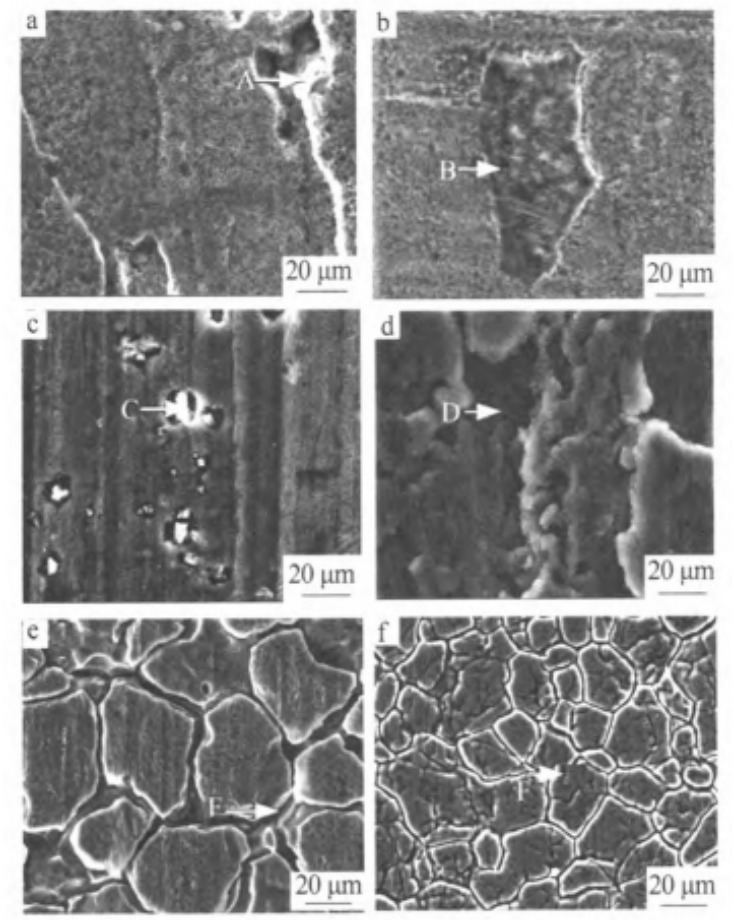

Fig. 3: SEM Picture of 6 Alloy Materials
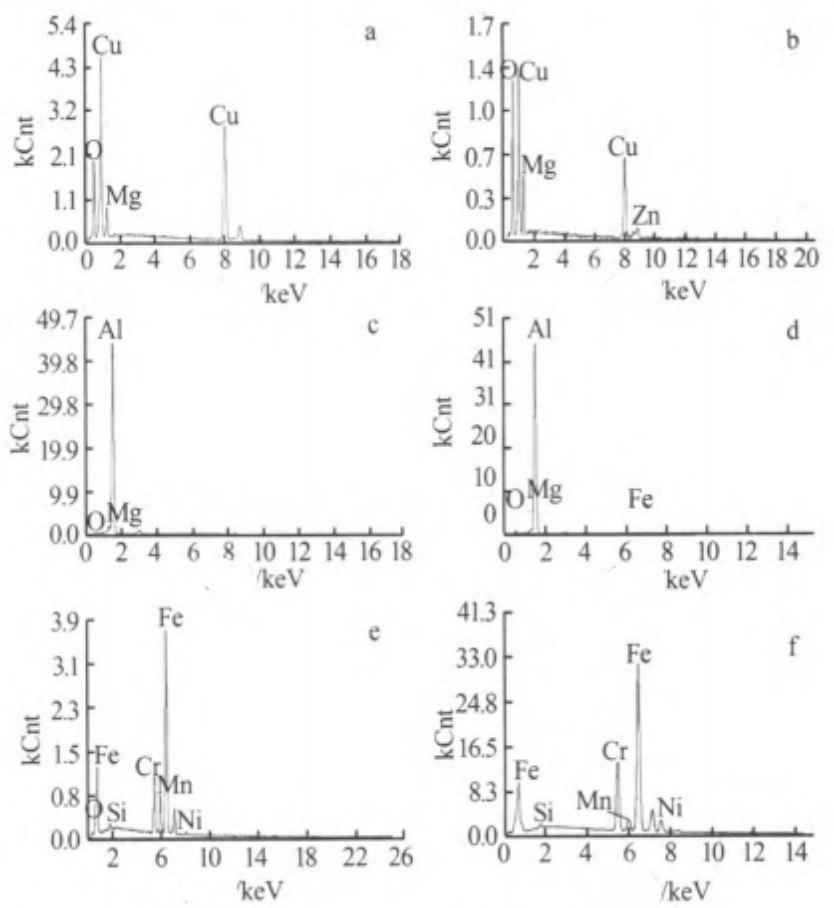

Fig. 4: Energy Spectrum Diagram of 6 Alloy Metals in PCMs

It can be observed from Figure 4a that components of red copper at A mainly include $\mathrm{O}, \mathrm{Cu}$ and Mg. Brass's obvious corrosion pit is B. Mass fraction of $\mathrm{Cu}, \mathrm{Zn}$ and $\mathrm{O}$ is $62.31 \%, 6.44 \%$ and 21.07\%, respectively, indicating that there is the dezincification corrosion and oxide of $\mathrm{Cu}$. The surface of 5052 aluminum alloy has multiple corrosion pits. The diameter of $\mathrm{C}$ is $10 \mu \mathrm{m}$. Main corrosion products are composed of $\mathrm{O}, \mathrm{M}$ and $\mathrm{Mg}$, indicating that corrosion products give priority to alumina. This is contrary to the results of XRD. On the contrary, the corrosion of 6063 aluminum alloy is evener. The attached corrosion products are loose and have irregular morphology. The 
corrosion products detected at $\mathrm{D}$ include $\mathrm{O}, \mathrm{Al}, \mathrm{Mg}$ and $\mathrm{Fe}$. It is proved that corrosion products are $\mathrm{Al}_{2} \mathrm{O}_{3}$, indicating that by comparing with 5052 aluminum alloy, the corrosion resistance is more excellent. Meanwhile, it can be found that 202 stainless steel surface has the intergranular corrosion, showing the corrosion products are some oxides. The 304 stainless steel surface only has the slight intergranular corrosion. $\mathrm{F}$ also only has the matrix element.

\section{Conclusions}

To sum up, the weight loss method is applied to test corrosion of 6 alloy materials, including red copper, brass, 5052 aluminum, 6063 aluminum, 202 stainless steel and 304 stainless steel, etc., in PCMs. The results show that stainless steel has the best corrosion resistance, but red copper has the worst corrosion resistance. The corrosion resistance of 6 alloy materials is ranked as: 304 stainless steel $>202$ stainless steel $>6063$ aluminum alloy $>5052$ aluminum alloy $>$ brass $>$ red copper. This lays a theoretical foundation on material selection of energy storage materials and corrosion prevention technology. As a result, brass, red copper and 5052 aluminum alloy shouldn't be applied in industrial applies, but 6063 aluminum alloy, 202 stainless steel and 304 stainless steel indeed are good choices, especially for 304 stainless steel of which lifetime can reach dozens of years.

\section{References}

[1] Fang Jueheng, Ke Xiufang and Shu Chang, Corrosion Behavior of Stainless Steel and Low Carbon Steel in High-concentration PCM Solution System[J], Guangdong Chemistry, 2014(7): 38-40;

[2] Tie Shengnian, Liu Xin and Tie Jian, Corrosivity of PCMs and Research Progress of Packaging Materials[J], Materials Review, 2015(11): 138-143;

[3] Shu Wenxiang and Guo Mingxing, et al., Corrosion Property Influence Factor Analysis of Al-Zn-Mg-(Cu) Alloy[J], Materials Review, 2013, 27(13): 1-9;

[4] Liu Jie, the Study on PCMs and Their Industrial Applications[J], Ceramics, 2014(2): 30-32;

[5] Liu Xin, Tie Jian and Tie Shengnian, the Study on Na2SO4·10H2O Composite Phase-Change Energy Storage Materials for Metal Packaging Materials[J], Journal of Intraocular Lens, 2016(4): 986-994;

[6] Wang Dan, Corrosion and Protection of Solar Thermal Power Plant in PCMs[D], Master's Thesis in Guangdong University of Technology, 2010;

[7] Zhang Yang and Li Yuefeng et al., The Research Overview of Thermal Stability and Container Compatibility in PCMs[J], Materials Review, 2011, 25(19): 18-23;

[8] Zhu Wenya, Yang Haitang and Zeng Dewen, The Corrosive Behavior Study on Alloy Materials in PCMs[J], Inorganic Salt Industry, 2015(8): 14-18;

[9] Zhang Helei, Fang Xiande and Zhao Yingjie, PCMs and Technical Research Progress[J], Materials Review, 2014, 28(13): 26-32;

[10] Xu Zhiguo, Zhao Changying and Ji Yunan, Research Progress of Middle and Low-temperature Phase Change Heat Storage[J], Energy Storage Science and Technology, 2014, 3(3): 31-35 